\title{
Study of Naval Ship Target Detection Probability of Skywave Radar based on Simplified Signal Processing Model
}

\author{
LIU Shihua ${ }^{1, a}$ XIANG Jiantao ${ }^{1, a}$ ZHU Yong ${ }^{2, a}$ \\ ${ }^{1}$ Dept.2, AFEWA, Wuhan 430019, China \\ ${ }^{2}$ MSR, AFEWA, Wuhan 430019, China \\ akyoliu2010@sina.com
}

\begin{abstract}
Keywords: sea clutter suppression, skywave radar, naval ship target, detection performance analysis

Abstract. The suppression ability of sea clutter suppression methods based on AR model, phase space reconstruction and are analyzed. The simulation analysis method of naval ship target detection performance based on sea clutter simulation data - sea clutter suppression method simplified signal processing] model is constructed. The ship target detection probability of skywave radar is researched after sea clutter suppression process by using above three sea clutter suppression methods.
\end{abstract}

\section{Introduction}

When the skywave radar detecting the naval ship target, the Doppler frequency is very small because the moving velocity of the large naval ship is very low. It is close to or submerged in the first order Bragg spectra of the sea clutter ${ }^{[1]}$. Therefore, the sea clutter add the difficulty to the naval ship target detection of the skywave radar.

According to the actual situation of the naval ship target detection of the skywave radar, at present, the study achievements of the sea clutter suppression method are obtained. The sea clutter suppression methods based on the empirical distribution stochastic models are presented. These methods are mainly include: (1) traditional methods ${ }^{[2-5]} ;$ (2) high resolution spectrum estimation $^{[6-8]}$; (3) orthogonal vector method ${ }^{[9]}$, phase space reconstruction method ${ }^{[10,11]}$. Using these methods, the naval ship target detection performance of skywave radar is getting better.

However, the present study is developed based on the sea clutter suppression methods, but there have few report of the skywave radar detection performance analysis after the sea clutter suppressed. In this paper, according to the actual situation of the skywave radar, a naval ship target detection performance analysis method is presented based on the simplified signal processing model, and the skywave radar naval ship target detection probability after the sea clutter suppression by the AR model, phase space reconstruction method and SVD method is computed.

\section{Naval Ship Target Detection Performance Analysis Method based on The Simplified Model}

The sea clutter is suppressed by the sea clutter suppression methods, meanwhile, the naval ship target detection performance of the skywave radar is improved. In order to analyze the naval ship target detection performance after sea clutter suppressing, the naval ship target detection performance analysis method of the skywave radar based on the simplified model is constructed as following. In this method, the naval ship target detection probability after sea clutter suppressing is studied.

In general, the signal processing in the sea target detection model of the skywave radar is include sea clutter suppression processing, coherent/incoherent accumulation and CFAR processing, as shown in figure 1. After signal accumulating, the target point is obtained by the CFAR processing with detection threshold. 


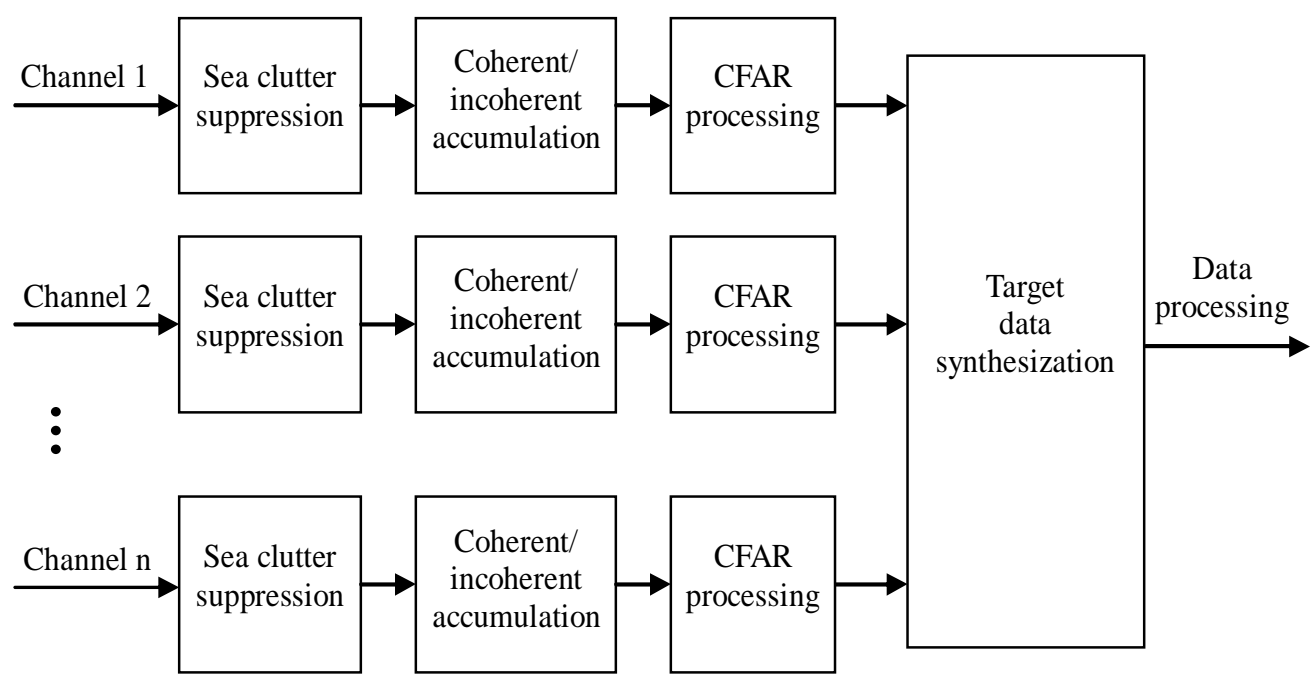

Figure 1 Principle block of signal processing subsystem

Because the signal precessing subsystem is very complicated, and the sea target detection model is under manufacturing, the useful determinate analysis method is not obtained. Based on the principle block of the signal processing subsystem, a simplified signal processing model of the skywave radar naval ship detection is presented, the processing flow chart of this model is shown as figure 2 .

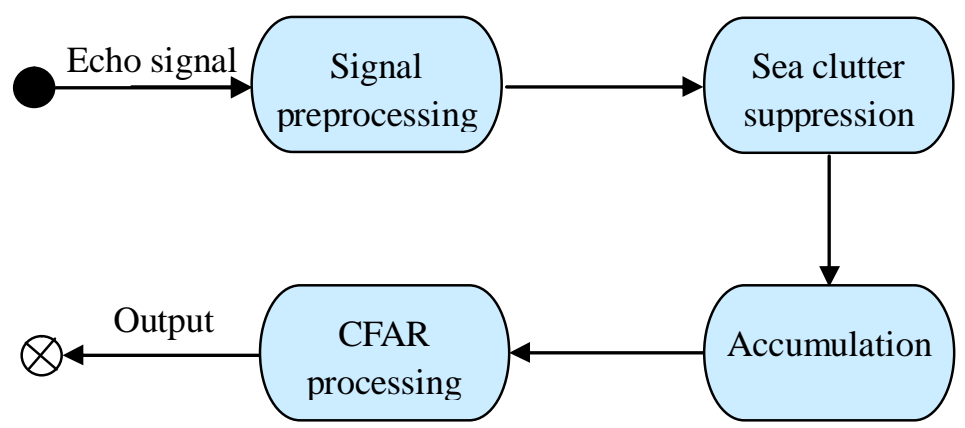

Figure 2 Processing flow chart of simplified signal processing model

(1) Signal preprocessing. The signal needed to be normalized before sea clutter suppressing to satisfied with the sea clutter suppression algorithms.

(2) Sea clutter suppression. The echo signal is processed by the sea clutter suppression algorithms. These algorithms include the AR model and the phase space reconstruction method.

(3) Coherent/incoherent accumulation. In ideal condition, the SCR of the echo signal is improved significantly after coherent/incoherent accumulation for several periods.

(4) CFAR processing. The traditional CFAR processing is mainly to detect the time domain enveloping of the echo signal, but the skywave radar is detect the target by the Doppler spectrum of the echo signal, so the range/Doppler 2-D CFAR detection method is needed. Based on the traditional CA-CFAR detection method, the modified range/Doppler 2-D CFAR samples selection12] and the detection processing flow chart are shown as figure 3 and figure 4 respectively. In figure 3 , the white cell $\mathrm{X}(\mathrm{fdi}, \mathrm{Rj})$ is detection resolution cell, the gray cells are the reference cells. In general, the number is $16-32$.

In the practical CFAR circuitry, based on the SNR index of the receiver, the CFAR threshold is needed to adjust dynamical to satisfy with the demand of Pd and Pf. The adjust coefficient $\mathrm{K}$ is decided by the false alarm probability of the CFAR detection.

The affection of the sea clutter suppression performance to the radar detection performance is reflected by the radar finding probability. In the engineering practice, the finding probability index is computed by the following: (1) Turn off the transmitter, keep the receiver working normally; (2) Adjusting the detection threshold under the given false alarm probability to satisfied with the demand of the false alarm probability; (3) Fixing the threshold to compute the finding probability. Obviously, this method is not appropriate for the equipments in developing. For this, a 
simplified method is analyzed. In order to discrimination, it is named "detection probability". The procedure of this method given by the following: (1) Simulating the sea clutter signal data with target; (2) Suppressing the sea clutter by the sea clutter suppression method; (3) Computing the detection performance by the above simplified signal processing method. For the consistence of the experiments, the sea clutter data include only one target, the evaluating value of the detection probability $\mathrm{P}_{\mathrm{d}}$ is

$$
\mathrm{P}_{\mathrm{d}}=N / M
$$

Where $N$ is the experiment times that the target can be detected, $M$ is the total experiment times.



Doppler

Figure 3 Range/Doppler 2-D CFAR samples selection block

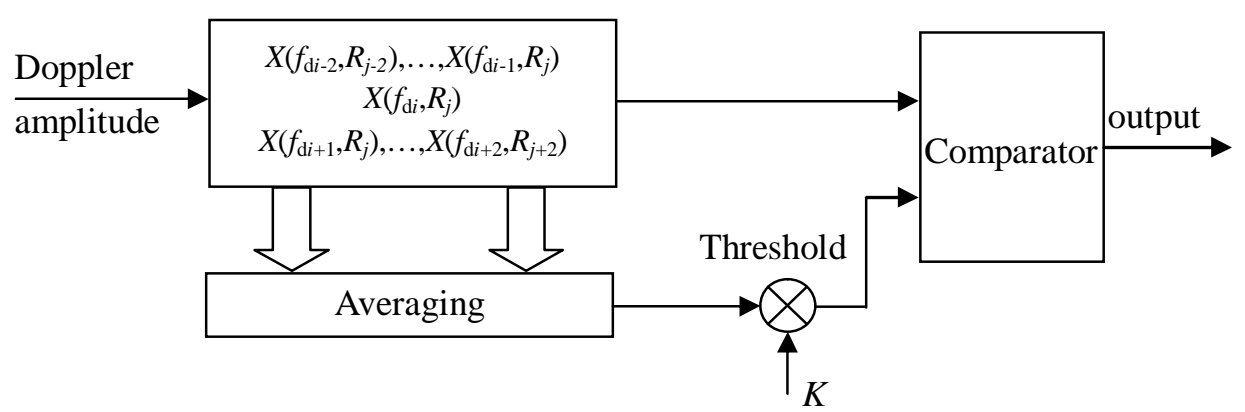

Figure 4 Range/Doppler 2-D CFAR detection processing flow chart

\section{Simulation and Analysis}

The sea clutter is suppressed by the AR model, the phase space reconstruction method and the SVD method. Let the SCR are $-5 \mathrm{~dB} 、-10 \mathrm{~dB}$ and $-15 \mathrm{~dB}$ respectively, the false alarm is 0.01 , the accumulation number $\mathrm{N}$ is are 1, 20 and 50 respectively, the number of the 2-D CFAR reference cells is 24 . The sea clutter signal is generated by simulation, there are different 100 groups data in each condition. After the sea clutter suppressing and the CFAR detection, the detection probabilities are shown in table 1 .

From the table 1 that we can conclude:

(1) The detection probabilities are very low without sea clutter suppression. In the most conditions, the naval ship can't be detected, the detection probabilities are 0. Even if the naval ship can be detected, the maximum detection probability is only 0.24 . The detection probability is not satisfied with our demand.

(2) The detection probabilities are significantly enhanced after sea clutter suppressing with the 
above three sea clutter suppression methods. Comparing these three methods, the detection performance of the phase space reconstruction method and the AR model are better than the SVD method. Otherwise, the computing time of the phase space reconstruction method and the AR model are less than SVD method. But, the phase space reconstruction method and the AR model need pure sea clutter data to construct sea clutter predictor, the applied condition is limited. Moreover, to compare with the AR model, the phase space reconstruction method can be used in the different sea states, but the AR model is simpler.

Table 1 Naval ship target detection probabilities of the skywave radar based on the simplified model

\begin{tabular}{|c|c|c|c|c|}
\hline $\begin{array}{l}\text { Suppres } \\
\text { sion } \\
F_{d}\end{array}$ & AR model & $\begin{array}{c}\text { Phase space } \\
\text { reconstructio } \\
\text { n }\end{array}$ & SVD & None \\
\hline \multicolumn{5}{|c|}{$\mathrm{SCR}=-5 \mathrm{~dB}, N=1$} \\
\hline $1 \mathrm{~Hz}$ & 0.7 & 1 & 0.16 & 0 \\
\hline $0.8 \mathrm{~Hz}$ & 0.1 & 0.99 & 0.12 & 0 \\
\hline $0.2 \mathrm{~Hz}$ & 0.01 & 0.9 & 0 & 0 \\
\hline \multicolumn{5}{|c|}{$\mathrm{SCR}=-10 \mathrm{~dB}, N=1$} \\
\hline $1 \mathrm{~Hz}$ & 0.03 & 0.92 & 0.11 & 0 \\
\hline $0.8 \mathrm{~Hz}$ & 0 & 0.83 & 0.02 & 0 \\
\hline $0.2 \mathrm{~Hz}$ & 0 & 0.65 & 0 & 0 \\
\hline \multicolumn{5}{|c|}{$\mathrm{SCR}=-15 \mathrm{~dB}, N=1$} \\
\hline $1 \mathrm{~Hz}$ & 0.01 & 0.78 & 0.04 & 0 \\
\hline $0.8 \mathrm{~Hz}$ & 0 & 0.64 & 0.04 & 0 \\
\hline $0.2 \mathrm{~Hz}$ & 0 & 0.57 & 0 & 0 \\
\hline \multicolumn{5}{|c|}{$\mathrm{SCR}=-5 \mathrm{~dB}, N=20$} \\
\hline $1 \mathrm{~Hz}$ & 1 & 1 & 0.31 & 0.1 \\
\hline $0.8 \mathrm{~Hz}$ & 1 & 1 & 0.21 & 0.02 \\
\hline $0.2 \mathrm{~Hz}$ & 1 & 1 & 0.02 & 0.01 \\
\hline \multicolumn{5}{|c|}{$\mathrm{SCR}=-10 \mathrm{~dB}, N=20$} \\
\hline $1 \mathrm{~Hz}$ & 1 & 1 & 0.3 & 0.03 \\
\hline $0.8 \mathrm{~Hz}$ & 0.69 & 1 & 0.14 & 0.01 \\
\hline $0.2 \mathrm{~Hz}$ & 0.49 & 0.99 & 0.03 & 0 \\
\hline \multicolumn{5}{|c|}{$\mathrm{SCR}=-15 \mathrm{~dB}, N=20$} \\
\hline $1 \mathrm{~Hz}$ & 0.94 & 0.99 & 0.23 & 0 \\
\hline $0.8 \mathrm{~Hz}$ & 0.11 & 0.99 & 0.09 & 0 \\
\hline $0.2 \mathrm{~Hz}$ & 0.05 & 0.94 & 0.01 & 0 \\
\hline \multicolumn{5}{|c|}{$\mathrm{SCR}=-5 \mathrm{~dB}, N=50$} \\
\hline $1 \mathrm{~Hz}$ & 1 & 0.99 & 0.4 & 0.24 \\
\hline $0.8 \mathrm{~Hz}$ & 1 & 0.99 & 0.35 & 0.11 \\
\hline $0.2 \mathrm{~Hz}$ & 1 & 0.99 & 0.1 & 0.1 \\
\hline \multicolumn{5}{|c|}{$\mathrm{SCR}=-10 \mathrm{~dB}, N=50$} \\
\hline $1 \mathrm{~Hz}$ & 1 & 1 & 0.28 & 0.07 \\
\hline $0.8 \mathrm{~Hz}$ & 1 & 1 & 0.18 & 0.04 \\
\hline $0.2 \mathrm{~Hz}$ & 0.96 & 1 & 0.05 & 0.01 \\
\hline
\end{tabular}




\begin{tabular}{|c|c|c|c|c|}
\hline \multicolumn{5}{|c|}{$\mathrm{SCR}=-15 \mathrm{~dB}, N=50$} \\
\hline $1 \mathrm{~Hz}$ & 1 & 1 & 0.31 & 0.02 \\
\hline $0.8 \mathrm{~Hz}$ & 0.63 & 1 & 0.21 & 0.01 \\
\hline $0.2 \mathrm{~Hz}$ & 0.4 & 0.99 & 0.04 & 0.01 \\
\hline
\end{tabular}

(3) When the SCR is same, the target Doppler spectra is more away from the Bragg spectra, the detection probability is higher. When the target is same, the SCR is bigger, the detection probability is higher.

(4) The accumulation can enhance the SCR, but the output is still not satisfied our demand. The sea clutter is accumulated when the target signal is accumulating. After the sea clutter suppression, the accumulation can significantly enhance the SCR. The detection performance of the phase space reconstruction method and the AR model are higher than the SVD method.

\section{Conclusion}

At present, the naval ship target detection pattern of the skywave radar is still developing, the detection performance is not computed by the existing methods. For this, a naval ship target detection performance simulation and analysis based on the simplified signal processing model is presented. From the simulation experiment we can conclude that the sea clutter suppression is significantly affected the detection performance of the skywave radar. The target can't be detected and the detection probability is very low without the sea clutter suppression. The detection probability is significantly improved after sea clutter suppressing. Furthermore, the detection probability is affected by the sea clutter suppression method, the SCR, the accumulation number and the target Doppler frequency.

\section{References}

[1] L. Yang. Study of skywave radar slow motion target detection technology in sea background[D]. Wuhan, Air Force Early Warning Academy, 2011

[2] B. Root. HF radar ship detection through clutter cancellation[C]. IEEE Radar Conference, 1998.

[3] G. Q. Qi, X. L. Jia. High accuracy frequency and phase estimation of single-tone based on phase of DFT[J]. Acta Electronica Sinica, 2001, 29(12): 1879-1882.

[4] J. B. Chen. Study of sea clutter suppression algorithm of high frequency skywave OTHR[D]. Shanghai, Shanghai Jiao Tong University, 2003.

[5] W. B. Xie, J. S. Lin. The geometric structure of nonlinear least square solution for signal by complex exponents and alternate algorithm[J]. Acta Electronica Sinica, 2002, 30(5): 757-759.

[6] J. L. Ying. Prediction of autoregressive Doppler signal based on Burg Alogrithm[J]. Science \& Technology Association Forum, 2010, (1): 59-60.

[7] C. M. Kang, Y. S. Yuan. The application of MUSIC method to detect the coherent sources on the background of ocean clutter[J]. Acta Electronica Sinica, 2004, 32(3): 502-504.

[8] Y. Fu, J. M. Liu, W. J. Lu. Design of PSS for excitation regulator with improved Prony algorithm[J]. Electric Power Automation Equipment, 2011, 31(4): 82-86.

[9] M. D. Xing, Z. Bao, Y. Qiang. Transient interference excision in OTHR[J]. Acta Electronica Sinica, 2002, 30(6): 823-826.

[10] Y. J. Tang. Ocean Clutter Suppression Using One-CLass SVM[C]. IEEE Workshop on Machine Learning for Signal Processing. 2004. 
[11]P. F. Yan, C. S. Zhang. Artificial neural network and simulated evolutionary computation[M]. Beijing: Tsinghua University Press, 2000.

[12]W. Y. Zhou, P. N. Jiao. Over-the-horizon radar technology[M]. Beijing: Publishing House of Electronics Industry, 2008. 